\title{
Analysis of the Performance of a Coherent SAC-OCDMA-OFDM-DWDM System Using a Flat Optical Frequency Comb Generator for Multiservice Networks.
}

Abderraouf Fares ( $\nabla$ faresabderraouf93@gmail.com)

Universite Badji Mokhtar de Annaba: Universite Badji Mokhtar Annaba https://orcid.org/0000-0002-9112-7060

Kaddour Saouchi

Universite Badji Mokhtar de Annaba: Universite Badji Mokhtar Annaba

Fatima Brik

Universite Badji Mokhtar de Annaba: Universite Badji Mokhtar Annaba

Hanane Djellab

University of Larbi Tebessi - Tebessa Faculty of Science and Technology: Universite Larbi Tebessi - Tebessa Faculte des Sciences et de la Technologie

\section{Research Article}

Keywords: Spectral Amplitude Coding Optical Code-Division Multiple-Access, Dense Wavelength Division Multiplexing, Orthogonal Frequency Division Multiplexing, Flat Optical Frequency Comb, Coherent Communication, Enhanced Double Weight, Random Diagonal, Bit Error Rate

Posted Date: January 3rd, 2022

DOI: https://doi.org/10.21203/rs.3.rs-1122460/v1

License: (c) (i) This work is licensed under a Creative Commons Attribution 4.0 International License. Read Full License 


\section{Abstract}

A SAC-OCDMA-OFDM-DWDM system using a flat optical frequency comb generator and a coherent communication system is proposed in this research. The objective is to develop a novel OCDMA communication system that is appropriate and dedicated to multiservice networks. A comparison between the two codes, EDW and RD, has been presented. It has been concluded that the RD code system has better performance in terms of robustness and number of users than the EDW code. By analyzing the BER for several symbol rates and comparing it to the pre-FEC threshold, the proposed system demonstrates its effectiveness against linear and nonlinear effects. Performance evaluation of the proposed system is carried out via co-simulation of OptiSystem and MATLAB.

\section{Introduction}

In recent years, optical code-division multiple-access (OCDMA) has emerged as one of the promising solutions as a multiple access technique for high capacity passive optical networks (PONs)(Mrabet et al. 2020; Kumawat and Maddila 2017; Kakaee et al. 2014; Seyedzadeh et al. 2017), due to the high demand for bandwidth, especially for 3D/4K/HD video streaming, e-learning and cloud computing applications. OCDMA architectures are suitable for Multiservice optical access networks, due mainly to their features such as full asynchronous multiple access capability, pliability in code design, low latency access, soft capacity on demand, high data confidentiality, and providing quality of service (QoS) differentiation at the physical layer(Tharek and Aljunid 2016). In addition, OCDMA has also gained attention as a robust and flexible system for free-space optical communications (FSO) which are suitable for $5 \mathrm{G}$ networks and their applications(Farghal 2019; Sarangal et al. 2017). Spectral amplitude coding OCDMA (SAC-OCDMA) is one of the various techniques developed for OCDMA systems(Upadhyay, Shukla, and Chaudhary 2019; Matem et al. 2019), because of its simplicity and lower cost of system components.

The conjunction of the OCDMA and Wavelength Division Multiplexing WDM system will limit the disadvantages of both techniques, such as the low security for wavelength and data modulated flow(Cao and Gan 2012; Singh and Singh 2017), and occurring inter-symbol interference (ISI) which leads to multiple access interference (MAl) that limits significantly the number of active users, therefore the hybridization of OCDMA/WDM allows easily the increase of channels' capacity and their level of confidentiality.

Orthogonal frequency division multiplexing (OFDM) has dominated wireless communication systems such as long-term evolution (LTE) networks, and also represents a future solution for $5 \mathrm{G}$ (Cai et al. 2018), because of its high spectral efficiency, robustness against delay, and increasing transmission rate through M-ary modulation of subcarriers like phase-shifted-keying (PSK) or quadrature amplitude modulation (QAM). In addition to the previously described advantages of the OCDMA/WDM system, the use of OFDM in this system provides robustness to fading and multipath channels and the ability to overcome the multi-access interface and multipath dispersion(Armstrong 2009), (Nawawi et al. 2018; Yousif et al. 2018), which reflect the specter efficiency.

Coherent optical communication is also seen as one of the promising solutions for PONs, because of the increase in the capacity of channels; providing the flexibility of wavelength selectivity and is very compatible and useful with long reach networks(Liu et al. 2010; Li and Dang 2017). The use of this technique in OCDMA systems will offer the same capacity as optical time-division multiple access (OTDMA) by multiplexing multiple users on the transmitted optical spectrum in order to suppress the presence of MAls in the asynchronous transmission mode.

In this paper, a SAC-OCDMA-OFDM-Dense WDM (DWDM) system dedicated to multiservice networks is proposed using a flat optical frequency comb (OFC) generator(Fortier and Baumann 2019) and coherent communication system. OCDMA codes are generated to be compatible and customized codes for multiservice networks; enhanced double weight (EDW) and random diagonal (RD) codes are mainly chosen due to their numerical properties, especially their low cross correlation characteristic(Abdullah et al. 2008; Fadhil et al. 2009) and their simple implantation. The bit error rate (BER) is the main parameter used to study and analyze the performance of the proposed system and verify which code can provide the maximum number of users and the most robust code against linear and nonlinear impairments. The paper is organized as follows: the second section presents a full description of the proposed system, then a numerical analysis of EDW and RD codes for coherent detection is shown in the third section, the simulation results are presented and discussed in the fourth section, and the conclusion is drawn in the fifth section.

\section{Proposed System}

The architecture of the proposed system is shown in figure 1. The SAC-OCDMA encoder for this system is a flat OFC generator, DWDM de-multiplexer, and optical power combiners, the SAC-OCDMA encoder is shown in figure 2. The flat OFC signal is generated by a continuous wave (CW) laser modulated with radio frequency (RF) signal using a dual-parallel Mach Zehnder modulator (DP-MZM)(Shang et al. 2015; Tran et al. 2019), assuming that the electric field of the CW laser can be expressed by:

$$
E_{C W l a s e r}=E_{0} \cos \left(\frac{2 \pi C}{\lambda_{0}} t+\phi_{0}\right)(1)
$$

Where $E_{0}$ is the amplitude of the electric field, Cis the speed of light, $\lambda_{0}$ is the CW laser wavelength, which is $1552.52 \mathrm{~nm}$ in this system and that is equivalent to $f_{0}=193.1 \mathrm{THz}$ and $\phi_{0}$ is the laser phase. Two RF signals are applied to the arms of the DP-MZM with the same frequency $f_{1}$ and with different voltages. The role of the DC bias voltages $V_{b_{1}}, V_{b_{2}}$ and $V_{b_{3}}$ is to control the transmission points of the DP-MZM. For the first arm, it is biased to the maximum transmission point by putting $V_{b_{1}}=0 \mathrm{~V}$, so the odd sidebands will be suppressed. In the second arm, $V_{b_{2}}$ has the same voltage as the switching bias voltage of the DP-MZM, so the transmission point of the second arm will be biased to the minimum transmission point in order to suppress the career and even sidebands. $V_{b_{3}}$ will be set to $0 \mathrm{~V}$, which means that $\phi_{3}$ will be zero, so phase shifting will be avoided at the output of the DP-MZM in order to get seven frequencies or wavelengths with spacing of $f_{1}$, the electric field of the DP-MZM output is:

Page 2/16 


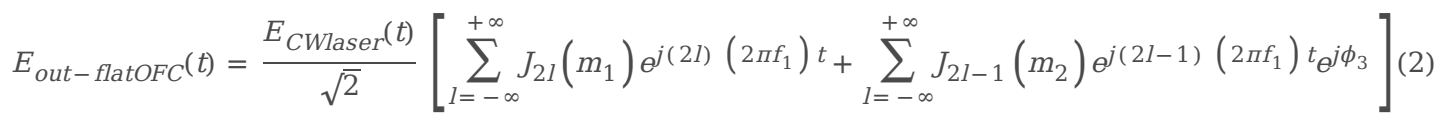

Where $J_{i}$ denotes the Bessel function of ith order of first kind, $m_{1}$ and $m_{2}$ are the modulation indexes of the first and second arm of the DP-MZM, $\phi_{3}$ is the bias angle. Figure 3 represents the Bessel function of first kind, the amplitude where there is an intersection of $J_{0}$ and $J_{2}$ is the same when $J_{1}$ and $J_{3}$ intersect, these two points mean that the DP-MZM can generate multiple optical sidebands creating a frequency comb, where $m_{1}=1.84$ and $m_{2}=3.05$, which means that six sidebands and the main career, all with the same amplitude, it can be expressed by:

$$
J_{-3}\left(m_{2}\right)=J_{-2}\left(m_{1}\right)=J_{-1}\left(m_{2}\right)=J_{0}\left(m_{1}\right)=J_{1}\left(m_{2}\right)=J_{2}\left(m_{1}\right)=J_{3}\left(m_{2}\right)(3)
$$

The modulation indexes are expressed as follows:

$$
\begin{aligned}
& m_{1}=\frac{\Pi R F 1}{V_{\Pi 1}}(4) \\
& m_{2}=\frac{\Pi R F 2}{V_{\Pi 2}}(5)
\end{aligned}
$$

Where $R F 1$ and $R F 2$ are the amplitudes of the RF signals, $V_{\Pi 1}$ and $V_{\Pi 2}$ are the half-wave voltages for each arm of the DP-MZM respectively. Figure 4 shows the CW laser and the flat OFC spectrums(Shang et al. 2015). In this system, $f_{1}$ is going to be $25 \mathrm{GHz}$, which is the equivalent of $\lambda_{1}=0.2 \mathrm{~nm}$. To split each wavelength to construct the SAC-OCDMA codes, a DWDM de-multiplexer is used for this purpose with spacing of $\lambda_{1}$, then a set of power combiners will be employed to combine the selected wavelengths to construct the SAC-OCDMA code for each user. Concerning the EDW and RD codes, the weight and the number of users have been fixed at three to get the same code length, which is six, so the code length of the EDW code $N_{E D W}$ is defined by the next expression(Menon et al. 2012; Abd El-Mottaleb et al. 2020):

$$
N_{E D W}=2 k+\frac{4}{3}\left[\sin \left(\frac{k \Pi}{3}\right)\right]^{2} \frac{8}{3}\left[\sin \left(\frac{(k+1) \Pi}{3}\right)\right]^{2}+\frac{4}{3}\left[\sin \left(\frac{(k+2) \Pi}{3}\right)\right]^{2}(6)
$$

Where $k$ is the number of users. The code length of the RD code is given by the next expression(Mostafa and Mohamed 2017; Upadhyay et al. 2019):

$$
N_{R D}=k+2 W-3(7)
$$

Where $W$ denotes the code weight. Table 1 and Table 2 show the EDW and RD codes for each user. The same number of users in this case leads to have the same MAI interferences, so the cross-phase modulation (XPM)(Tithi and Majumder 2020) has almost the same effect on both signals during the transmission. Figure 5 represents the EDW and the RD spectrums.

Table 1

EDW codes for $W=3, N_{E D W}=6$
\begin{tabular}{|lllllll|}
\hline User \#1 & $\mathbf{0}$ & $\mathbf{0}$ & $\mathbf{1}$ & $\mathbf{1}$ & $\mathbf{0}$ & $\mathbf{1}$ \\
\hline User \#2 & 0 & 1 & 0 & 0 & 1 & 1 \\
\hline User \#3 & 1 & 1 & 0 & 1 & 0 & 0 \\
\hline
\end{tabular}

Table 2

RD codes for $W=3, N_{R D}=6$

\begin{tabular}{|lllllll|}
\hline User \#1 & $\mathbf{0}$ & $\mathbf{0}$ & $\mathbf{1}$ & $\mathbf{0}$ & $\mathbf{1}$ & $\mathbf{1}$ \\
\hline User \#2 & 0 & 1 & 0 & 1 & 1 & 0 \\
\hline User \#3 & 1 & 0 & 0 & 1 & 0 & 1 \\
\hline
\end{tabular}

Since the length of both codes is six, just 6 successive sidebands will be selected for the coherent modulation. After the code construction, the code's spectrum will be modulated with an OFDM signal using an optical coherent modulator as shown in the figure 5. Pseudo random binary sequence generator is employed as user data, then a mapping system is used to generate the in-phase and the quadrature components sequences, in this system the QPSK and 16-QAM modulation will be employed.

After separating the I and the Q components, the OFDM modulator will be employed. After the modulating the two components, the general electrical OFDM generated signal with $N_{S C}$ subcarriers in the $k_{t h}$ symbol period can be expressed as(Chen et al. 2014):

$$
s_{k}(t)=\sum_{n=0}^{N_{s c}-1} C_{k, n} e^{j \frac{2 \pi n t}{T}(8)}
$$


Where $C_{k, n}$ designates the complex coefficient on the $n_{t h}$ subcarrier in the $k_{t h}$ symbol and $T$ is the OFDM symbol time. The OFDM modulator parameters are: The number of subcarriers is 512 and the IFFT points $=1024$ with a cyclic prefix of the transmitted symbol. The $I(t)$ and $Q(t)$ of the electrical OFDM signal will be modulated with the SAC-OCDMA user code, each OFDM component will be modulated by a dual drive Mach-Zehnder modulator (DD-MZM).

Both of the DD-MZMs are biased to the null transmission point due to its minimizing of the radio to optical up-converter nonlinearities(Shieh et al. 2008). $V_{b} / 2$ is the bias voltage where $V_{b}$ is the switching bias voltage of the DD-MZMs. After the modulation, the optical $Q(t)$ component doesn't need an optical phase shifter since the phase is shifted in the OFDM modulator(Sheetal and Singh 2018), so both of the components will be combined using a power combiner. The output of the coherent optical OFDM modulator represents one user, to combine all the users, other power combiners are used for this purpose, which can be expressed by:

$$
E_{S}(t)=\sum_{k=1}^{N_{u}} A_{I k} I_{k}(t) e^{j 2 \Pi f_{k} t+j \phi_{k}}+A_{Q k} Q_{k}(t) e^{j 2 \pi f_{k} t+\frac{\pi}{2}+j \phi_{k}(9)}
$$

Where $N_{u}$ is the number of users, $A_{I k}$ and $A_{Q k}$ are the OCDMA encoded signals amplitudes of each component for each user, which are proportional to the I and Q components, respectively. Modulation indexes and the phase shifts of the DD-MZMs, $I_{k}(t)$ and $Q_{k}(t)$ are the OFDM I/Q components for each user(Shieh et al. 2008), $f_{k}$ is set of frequencies that identify and $\phi_{k}$ is the phase for each user. Figure 6 shows the EDW and the RD output spectrums.

After the transmission of the combined signals, they will be decoded by splitting each wavelength alone using a DWDM de-multiplexer. Then the same set of power combiners employed in the SAC-OCDMA encoder is used in order to construct each user spectrum for the second time. Figure 7 shows the SAC-OCDMA decoder. Coherent detection is the technique that will be used to restore the OFDM signal. Furthermore, the local oscillator (LO) is mandatory for this technique. In this proposed system, the LO part will be a second flat OFC generating seven wavelengths with spacing of $\lambda_{1}$, then another DWDM de-multiplexer used for the purpose of splitting each wavelength, then a selection of wavelengths that are the same as the received signal that do not contain spectral interference, and putting each selected wavelength which represents the users and the received signal into the inputs of the $90^{\circ}$ hybrid coupler in the receiver of each user.

Figure 8 shows the optical coherent detector including a $90^{\circ}$ hybrid coupler and two balanced detectors for $I(t)$ and $Q(t)$ components recovering (Painchaud et al. 2009). The output electric fields of the $90^{\circ}$ hybrid coupler after mixing the received signal with the LO signal are written:

$$
\begin{aligned}
& E_{1}(t)=\frac{1}{\sqrt{2}}\left[E_{S}(t)+E_{L O}(t)\right](10) \\
& E_{2}(t)=\frac{1}{\sqrt{2}}\left[E_{S}(t)-E_{L O}(t)\right](11) \\
& E_{3}(t)=\frac{1}{\sqrt{2}}\left[E_{S}-j E_{L O}(t)\right](12) \\
& E_{4}(t)=\frac{1}{\sqrt{2}}\left[E_{S}(t)+j E_{L O}(t)\right](13)
\end{aligned}
$$

Where $E_{S}(t)$ and $E_{L O}(t)$ are the electric fields of the received signal and the LO signal, the received signal contains six modulated signals three of them are the interference spectrums, it can be assumed by the next expression:

$$
E_{S}(t)=A_{s 1}(t) e^{j\left(\omega_{1} t+\phi_{1}\right)}+A_{s 2}(t) e^{j\left(\omega_{2} t+\phi_{2}\right)}+A_{s 3}(t) e^{j\left(\omega_{3} t+\phi_{3}\right)}+A_{s 4}(t) e^{j\left(\omega_{4} t+\phi_{4}\right)}+A_{s 5}(t) e^{j\left(\omega_{5} t+\phi_{5}\right)}+A_{s 6}(t) e^{j\left(\omega_{6} t+\phi_{6}\right)}(14)
$$

And the LO signal can be expressed:

$$
E_{L O}(t)=A_{L O} e^{j\left(\omega_{L O} t+\phi_{L O}\right)}(15)
$$

Where the square of $A_{s 1}(t), A_{s 2}(t), A_{s 3}(t), A_{s 4}(t), A_{s 5}(t)$ and $A_{s 6}(t)$ are the powers for each wavelength, and the square of $A_{L O}$ is the power of the $L O$ signal. After mixing the received signal with the LO signal and detecting the electric signal using a balanced detector, assuming the all phases are equal, the output photocurrents can be given by :

$\Delta i_{I}(t)=R A_{L O}\left[A_{s 1}(t) \cos \left(\omega_{I F 1} t\right)+A_{s 2}(t) \cos \left(\omega_{I F 2} t\right)+A_{s 3}(t) \cos \left(\omega_{I F 3} t\right)+A_{s 4}(t) \cos \left(\omega_{I F 4} t\right)+A_{s 5}(t) \cos \left(\omega_{I F 5} t\right)+A_{s 6}(t) \cos \left(\omega_{I F 6} t\right)\right](1$

$\Delta i_{Q}(t)=R A_{L O}\left[A_{s 1}(t) \sin \left(\omega_{I F 1} t\right)+A_{s 2}(t) \sin \left(\omega_{I F 2} t\right)+A_{s 3}(t) \sin \left(\omega_{I F 3} t\right)+A_{s 4}(t) \sin \left(\omega_{I F 4} t\right)+A_{s 5}(t) \sin \left(\omega_{I F 5} t\right)+A_{s 6}(t) \sin \left(\omega_{I F 6} t\right)\right](17)$

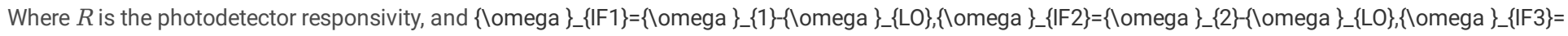

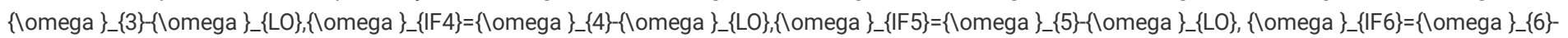
$\{$ lomega $\}\{$ LO $\}$,

Knowing that $\{\backslash \text { omega }\}_{-}\{\mathrm{LO}\}$ is the same as one of the six wavelengths which is the selected spectrum to be demodulated, that means that $\{\backslash \text { lomega }\}_{-}\{\mid \mathrm{F}\}$ is going to be zero and the selected spectrum will be amplified due to its mixing with the LO signal and shifted to become a baseband signal. Figure 9 shows the balanced detector output spectrums of both codes. 
The received signal components will be filtered using low pass cosine roll-off filters with a roll-off coefficient of 0.2 in order to keep the baseband part of the received signal and eliminate all the undesirable frequencies, and minimize the inter-symbol interferences in the OFDM signal. Then an OFDM demodulator, QPSK or 16-QAM decoder and digital signal processing (DSP) are used to restore the original transmitted signal, observe and study the system performance. The DSP is used generally with analog-to digital converters, its role is mainly to compensate for digitally the channel impairments such as chromatic dispersion (CD), polarization mode dispersion (PMD)(Amari et al. 2017), and all phase noises using adaptive equalizers by removing the rate of rotation of the constellation using finite impulse response filters. The DSP employs frequency offset estimator and career phase recovery algorithms for recovering the transmitted signal.

\section{Mathematical Analysis}

The theoretical performance of the EDW and RD codes of the optical coherent detection system will be analyzed. The signal to noise ratio (SNR) of the received signal determines the estimated BER value, the theoretical SNR is expressed by:

$\mathrm{SNR}=\backslash$ frac $\left\{\{\mid\}^{\wedge}\{2\}\right\}\left\{\{\backslash \text { sigma }\}^{\wedge}\{2\}\right\} \backslash$ left(18\right)

Where $\left\{\{\}^{\wedge}\{2\}\right.$ represents the power spectral density of the received photocurrent, and $\{\backslash \text { sigma }\}^{\wedge}\{2\}$ is the variance of the noise sources in the transmission system. In optical coherent detection, the main noise sources are thermal noise, shot noise and the amplified spontaneous emission noise (ASE noise). The intensity noise is neglected in this work because the intensity noise of the transmitted signal is eliminated by the transmission media high losses and the LO intensity noise is eliminated by balanced detection(Buscaino et al. 2019). Before detailing each noise, the transmitted signal average power should be detailed in order to determine the received photocurrent and the noise power. In the optical coherent detection described in the previous section, a $90^{\wedge} \backslash$ circ hybrid coupler is used to permit the splitting of the in-phase I and quadrature Q components of the transmitted signal which is modulated by a coherent modulator. Knowing that the $\mathrm{Q}$ component is phase shifted by $90^{\wedge} \backslash \mathrm{circ}$, in order to simplify our analysis, the I component will be considered because there is no phase shifting. The root mean square of the received photocurrent of the I component in the output of the $90^{\wedge}$ \circ hybrid coupler is:

$\{\mid\}_{-}\{1\}=\mathrm{R} \backslash$ sqrt $\left\{\left\{(\mathrm{P}\}_{-}\{r\}\{\mathrm{P}\}_{-}\{\mathrm{LO}\} / 4\right)\right\} \backslash$ left(19\right)

Where $\{P\}_{-}\{r\}$ and $\{P\}_{-}\{L O\}$ are the received power of the received signal and the LO power in the receiver. The received signal is an OCDMA encoded signal. In order to get the received electrical signal using coherent detection ( $90^{\wedge} \backslash$ circ hybrid coupler and balanced detector), the signal power of EDW and RD codes are defined by their mathematical proprieties. From (Abdullah et al. 2008), the EDW code average power for spectral direct detection (SDD) is defined by the following expressions:

\section{$\{P\}_{-}\{E D W\}=P W / N \backslash$ left(20\right)}

Where $\mathrm{P}$ is the signal effective power at the receiver, $\mathrm{W}$ denotes the code weight, and $\mathrm{N}$ represents the code length. From (19) and (20), the photocurrent of the I component for EDW code is expressed by the next formula:

$\{\mid\}_{-}\left\{\{\mid\}_{-}\{E D W\}\right\}=R \backslash$ sqrt $\left\{\backslash\right.$ frac $\left.\left\{\left\{\{W P\}_{-}\{r\}\right\}_{-}\{E D W\}\{P\}_{-}\{L O\}\right\}\left\{4\{N\}_{-}\{E D W\}\right\}\right\} \backslash$ left(21\right)

Concerning the RD code, according to (Fadhil et al. 2009), the power of the RD code for SDD detection is defined by the next expression:

$\{P\}_{-}\{R D\}=2 P W / N \backslash$ left(22\right)

By replacing (22) in (19), the photocurrent of the RD code signal is:

$\{\mid\}_{-}\left\{\{\mid\}_{-}\{R D\}\right\}=R \backslash$ sqrt $\left\{\backslash\right.$ frac $\left.\left.\left\{\left\{\{W P\}_{-}\{r\}\right\}_{-}\{R D\} P P\right\}_{-}\{L O\}\right\}\left\{2\{N\}_{-}\{R D\}\right\}\right\} \backslash$ left(23\right)

Consequently, the variance of the noise sources can be determined; we can express it by the next formula:

$\{\backslash \text { sigma }\}^{\wedge}\{2\}=\left\{\{\backslash \text { sigma }\}^{\wedge}\{2\}\right\}_{-}\{$shot $\}+\left\{\{\backslash \text { sigma }\}^{\wedge}\{2\}\right\}_{-}\{\text {ASE }\}_{+}\left\{\{\backslash \text { sigma }\}^{\wedge}\{2\}\right\}_{-}\{$thermal $\} \backslash$ left(24\right } )

Where $\left\{\{\backslash \text { sigma }\}^{\wedge}\{2\}\right\}_{-}\{$shot $\}$is the shot noise, $\left\{\{\backslash \text { sigma }\}^{\wedge}\{2\}\right\}_{-}\{$ASE $\}$is the ASE noise and $\left\{\{\backslash \text { sigma }\}^{\wedge}\{2\}\right\}_{-}\{$thermal $\}$is the thermal noise. In this analysis, the shot noise of the LO in the receiver is the dominant noise, and as described in the previous section, the LO signal contains only the selected wavelength that will be recovered from the transmitted signal. This noise can be modeled by the next variance formula:

$\left\{\{\backslash \text { sigma }\}^{\wedge}\{2\}\right\}_{-}\{$shot $\}=q\{P\}_{-}\{$LO $\} R B \backslash$ left(25\right } )

Where $\mathrm{q}$ is the electron's charge and B is the effective bandwidth of the photodetector's receiver. In regards to ASE noise, the employing of inline optical amplifiers that spontaneously generates noise that degrade the quality of the received signal. In this analysis, the local oscillator-ASE beating noise is the dominant ASE noise. It can be expressed by:

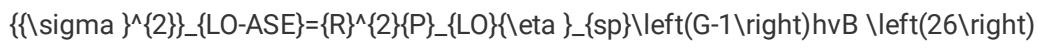

Where $\{\text { leta }\}_{-}\{\mathrm{sp}\}$ is the spontaneous emission factor, $\mathrm{G}$ is the amplifier gain, $\mathrm{h}$ is the Planck's constant and $\mathrm{v}$ is the photon's frequency. The thermal noise of EDW is the same as the RD code because it is always independent of the received photocurrent. The expression of the thermal noise is :

$\left\{\{\backslash \text { sigma }\}^{\wedge}\{2\}\right\}_{-}\{$thermal $\}=\backslash$ frac $\left\{4\{K\}_{-}\{B\}\{T\}_{-}\{n\} B\right\}\left\{\{R\}_{-}\{L\}\right\} \backslash$ left(27\right } ) 
$\{K\}_{-}\{B\}$ is Boltzmann's constant, $\{T\}_{-}\{n\}$ is the absolute receiver noise temperature, and $\{R\}_{-}\{L\}$ is the receiver load resistor. After defining all the currents and the noise, the SNR can be calculated for the EDW and RD codes by the following expressions:

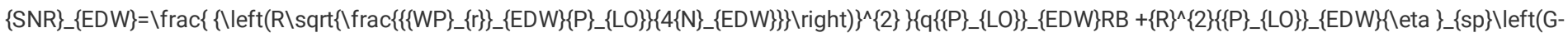
$1 \backslash$ right $\left.) h v B+\backslash f r a c\left\{4\{K\}_{-}\{B\}\{T\}_{-}\{n\} B\right\}\left\{\{R\}_{-}\{L\}\right\}\right\} \backslash$ left(28\right)

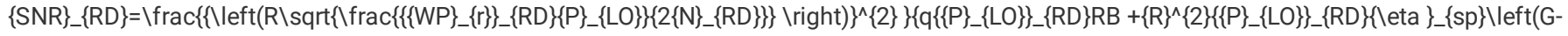

$1 \backslash$ right)hvB+\frac $\left.\left\{4\{K\}_{-}\{B\}\{T\}_{-}\{n\} B\right\}\left\{\{R\}_{-}\{L\}\right\}\right\} \backslash$ left(29\right)

In order to have the most direct measure which to help to study the performance of both codes in an optical coherent detection system, the estimated BER is calculated by the following formula(Mrabet et al. 2018):

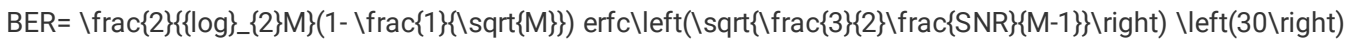

$\mathrm{M}$ is the high order of the advanced modulation format (M-PSK or M-QAM), since the transmitted data uses these mapping types to be employed in OFDM modulation.

Analytically, to study the performance of the EDW and RD codes for optical coherent detection, the number of active users should be defined and determined which code may provide the maximum number of users, the BER is the main parameter that could judge which code is the best. To calculate the BER, the code length will be defined mainly as a function of the number of users $k$, it can be calculated from \left(6\right) and \left(7\right).

The number of users is shown in Figure 10 of the SAC-OCDMA coherent detection system for the EDW and RD codes using 16-QAM-OFDM modulation for different code weights and different bit rates in the presence of ASE noise. Clearly, the RD supports a larger number of active users for different code weights and different bit rates due to its low cross correlation characteristic. The MAl presence in the transmitted signal spectrum is reduced compared to the EDW code, which its suppression is simple and easier to implement. In addition, the presence of ASE noise decreases sharply the number of users due to its effects on the received signal, so the use of the inline amplifiers in this system is not recommended.

However, in this system, the RD code cannot be considered better than the EDW code in terms of robustness against linear and nonlinear impairments, the performance should be studied when the number of users and the code weight are equal for each code, in order to find out which code is performing better.

\section{Simulation Results And Discussion}

Besides the aim of studying the performance of the proposed system, co-simulation technique is employed through the use of OptiSystem and MATLAB. Furthermore, the optical system is carried on the OptiSystem, the running of the simulation and the processing of the results as well as its presentation which is the task of MATLAB. The BER will be calculated from the measured EVM from the constellation diagram. Moreover, the EVM provides an accurate measurement to evaluate multi-level and multi-phase modulation systems such as QPSK and 16-QAM modulations. Thus, it can be defined as the difference between the reference symbol vector and the measured symbol vector illustrated in the constellation, it can be expressed by(Schmogrow et al. 2012):

$E V M=\backslash$ sqrt $\left\{\right.$ frac $\left\{\{P\}_{-}\{\right.$err $\left.\}\right\}\left\{\{P\}_{-}\{\right.$ref $\left.\left.\}\right\}\right\} \backslash$ left(31\right)

Where $\{P\}_{-}\{$err $\}$is the average power of the error vector of the received data, including all the linear and nonlinear impairments, $\{P\}_{-}\{$ref $\}$is the average power of the reference symbol vector.

In this simulation, the optimum CW laser injected power into the transmitter and into the LO in the receiver for each user has been defined by varying the injected power and determining which power corresponds to the minimum EVM in order to minimize the optical fiber nonlinear effects specifically the Kerr's effect and the self-phase modulation (SPM), and getting sufficient power for the transmission. Figure 11 shows that for both of codes, EDW and RD, the minimum EVM corresponds to an injected power of $10 \mathrm{dBm}$ for symbol rate of $10 \mathrm{GBd}$ using QPSK modulation over $60 \mathrm{Km}$ including $50 \mathrm{Km}$ of single-mode fiber (SMF), $10 \mathrm{Km}$ of dispersion compensating fiber (DCF) and an erbium-doped fiber amplifier (EDFA) for attenuation compensation(Sheetal and Singh 2018). The figure also shows that the RD code performs better than the EDW when the injected power is varied.

The influence of the noise presence will be studied by adding ASE noise to the transmitted signal and evaluating the performance of the system for both codes. Moreover, the optical signal-to-noise ratio (OSNR) is the optical power divided by the ASE noise, the OSNR is measured over a reference OSNR where its ASE noise bandwidth is $0.1 \mathrm{~nm}$ at $1550 \mathrm{~nm}$, by varying the ASE noise, the OSNR will also be also varied, which will influence the performance of the EVM, the BER can be calculated using the following expression(Freude et al. 2012; Schmogrow et al. 2012):

$$
B E R=\frac{1-M^{-1 / 2}}{\frac{1}{2} \log _{2} M} \operatorname{erfc}\left(\sqrt{\frac{3 / 2}{(M-1) r^{2} E V M^{2}}}\right)
$$

The constant $r$ is depending on the modulation format, from (Freude et al. 2012), $\{r\} \_\{Q P S K\}=1$ and $\{r\} \_\{16-Q A M\}=\mid s q r t\{\backslash$ frac $\{9\}\{5\}\}$.

Figure 12 represents the $\log (\mathrm{BER})$ in function of the OSNR for symbol rate of $10 \mathrm{GBd}$ for each user over several distances, which are: back-to-back (BtB) transmission, $50 \mathrm{Km}$ using SMF and $60 \mathrm{Km}$ which was described previously, and comparing its performance with pre-forward error correction (pre-FEC) threshold of 2.17\times $\{10\}^{\wedge}\{-3\}$ in order to find out if the transmitted data can be recovered(Agrell and Secondini 2018), from Fig. 12 (a), all the curves are below the pre-FEC threshold when the QPSK modulation is employed when the OSNR starts from $5 \mathrm{~dB}$. However for the performance 16-QAM modulation 
shown in Fig. 12 (b), the threshold is respected after $9 \mathrm{~dB}$ for BtB transmission for both codes, $10 \mathrm{~dB}$ for $60 \mathrm{Km}$ for both codes too, almost $11 \mathrm{~dB}$ for RD code and EDW code for $50 \mathrm{Km}$. Figure 13 shows the log(BER) versus the OSNR for the distance of $60 \mathrm{Km}$ described before, Fig. 13 (a) shows that the symbol rate of $15 \mathrm{GBd}$ for each user, starting from $7 \mathrm{~dB}$ the QPSK system, is also below the pre-FEC threshold for both codes. Concerning the 16-QAM modulation, the EDW and RD codes verified the threshold at almost $12 \mathrm{~dB}$. For symbol rate of $20 \mathrm{GBd}$ for each user shown in Fig. 13 (b), the pre-FEC threshold is verified for higher OSNR. Concerning the QPSK modulation, the EDW code respects the threshold at almost $7 \mathrm{~dB}$, and the RD code is still below the pre-FEC threshold. The threshold is also respected for OSNR of $15 \mathrm{~dB}$ and $17 \mathrm{~dB}$ for the RD and EDW codes respectively.

With regard to the discussion of the obtained results, due to the DWDM system used in this proposed system, the XPM is induced from DWDM adjacent wavelengths, which specifically results nonlinear phase noise due to the cumulative intensity dependence of the refractive index in the optical fiber, which heavily distorts the received signal. It is very clear in all figures 11, 12 and 13 that the RD code system performs better than the EDW code for all the cases studied. Furthermore, the presence of wavelengths interferences takes place at the extremity of the RD code spectrum as shown in Fig. 6 (b). On the other hand, for the EDW code, the interferences are located in the vicinity of the users wavelengths as shown in Fig. 6 (a). Moreover, the overlap of adjacent spectrums results in the XPM effect with interfered spectrums, which degrades the quality of detected signals. However for the RD code, the overlap is limited to the interfered spectrums due to their positions at the extremity of the transmitted spectrums.

Figure 14 shows the constellation diagrams of both EDW and RD codes of the third user for a symbol rate of $20 \mathrm{GBd}$ for BtB transmission, $120 \mathrm{Km}$ using twice the $60 \mathrm{Km}$ optical link which was described previously \left(\right(50 Km-SMF + $10 \mathrm{Km}-\mathrm{DCF}+$ EDFA)\times 2), and $300 \mathrm{Km}$ using the same optical link five times $\backslash$ left(\right(50 Km-SMF + $10 \mathrm{Km}$-DCF + EDFA)\times 5). The IQ skew highly apparent in the BtB transmission due mainly to the electrical generation of the I left(t\right) and Q $\backslash$ left(t\right) which are not separated exactly by $90^{\wedge} \backslash$ circ, which distorts the received signal. For 300 Km QPSK system, the quality of the received signal constellation using RD code is better than the EDW. The same thing is observed for the constellation of 16-QAM system for a distance of 120 $\mathrm{Km}$, where the received symbols spread out clearly in the EDW code more than the RD code because of the influence of overlapping discussed already.

The RD code performs better than the EDW thanks to the low cross correlation characteristic which provides the maximum number of users. The localization of the interferences in the received signal demonstrates the robustness of the RD against the optoelectronic noise and the nonlinear effects of the optical fiber for coherent communication system.

\section{Conclusion}

In this paper, a coherent SAC-OCDMA-OFDM-DWDM system dedicated to multiservice networks has been studied, which employs a flat OFC generator for code generation in the transmitter, and is also used in the LO of the receiver in order to decode the received signal. The DWDM system is also employed for code construction because of the frequency used in the flat OFC generator and its accurate selection of each wavelength. Then, after applying the most known SACOCDMA codes, EDW and RD to this proposed system to see the performance of its detection technique, the latter shows its effectiveness against linear and nonlinear effects by analyzing the BER for several symbol rates and comparing it with the pre-FEC threshold, mainly due to the conjunction of SAC-OCDMAOFDM with the DWDM system despite the XPM and its influence on the performance of the transmitted signals.

\section{Declarations}

\section{Funding}

The authors declare that no funds, grants, or other support were received during the preparation of this manuscript.

\section{Competing interests/ Conflicts of interest}

The authors have no relevant financial or non-financial interests to disclose. The authors also declare that they have no conflict of interests.

\section{Authors' contributions}

Kaddour Saouchi and Fatima Brik supervised the project. The development of the idea, its design and the analysis of the study were performed by Abderraouf Fares, and Hanane Djellab. The first draft of the manuscript was written by Abderraouf Fares and all authors commented on previous versions of the manuscript. All authors read and approved the final manuscript.

\section{Availability of data and materials}

The datasets generated during and analyzed during the current study are available from the corresponding author on reasonable request.

\section{Code availability}

In the present work, the designed system is numerically simulated and analyzed using the OptiSystem. MATLAB is employed to present the results.

\section{Acknowledgements}

Not applicable.

\section{References}


1. Abd El-Mottaleb, S. et al.: MDW and EDW / DDW Codes with AND Subtraction / Single Photodiode Detection for High Performance Hybrid. Optical and Quantum Electronics 52(2020). https://doi.org/10.1007/s11082-020-02357-x.

2. Agrell, E., Secondini, M.: Information-Theoretic Tools for Optical Communications Engineers. 2018 IEEE Photonics Conference (IPC) (2018). https://doi.org/10.1109/IPCon.2018.8527126.

3. Amari, A., Octavia, A.D., Ramachandran, V., Sunish, K.O.S., Philippe C., and Yves, J.: A Survey on Fiber Nonlinearity Compensation for 400 Gbps and beyond Optical Communication Systems. IEEE Communications Surveys \& Tutorials 19 (4): 1-17(2017). https://doi.org/10.1109/COMST.2017.2719958.

4. Armstrong, J.: OFDM for Optical Communications. Journal of Lightwave Technology 27 (3): 189-204(2009). https://doi.org/10.1109/JLT.2008.2010061.

5. Buscaino, B., Brian, D.T., and Kahn, M.J.: Multi-Tb / S-per-Fiber Coherent Co-Packaged Optical Interfaces for Data Center Switches. Journal of Lightwave Technology 37 (13): 3401-12 (2019). https://doi.org/10.1109/JLT.2019.2916988.

6. Cai, Y., Zhijin, Q., Fangyu, C., Li, G.Y., Mccann, J.A.: Modulation and Multiple Access for $5 \mathrm{G}$ Networks. IEEE Communications Surveys \& Tutorials 20 (1): 629-46 (2018). https://doi.org/10.1109/COMST.2017.2766698.

7. Cao, Y., Gan, C.: A Scalable Hybrid WDM / OCDMA-PON Based on Wavelength-Locked RSOA Technology. Optik - International Journal for Light and Electron Optics 123 (2): 176-80 (2012). https://doi.org/10.1016/j.jjleo.2011.03.015

8. Chen, C., Zhong, W.: MDPSK Based Non - Equalization OFDM for Coherent Free - Space Optical Communication. IEEE Photonics Technology Letters 1135 (c): 1-4 (2014). https://doi.org/10.1109/LPT.2014.2329133.

9. Fadhil, H.A., Aljunid, S.A., Ahmad, R.B.: Optical Fiber Technology Performance of Random Diagonal Code for OCDMA Systems Using New Spectral Direct Detection Technique. Optical Fiber Technology 15 (3): 283-89 (2009). https://doi.org/10.1016/j.yofte.2008.12.005.

10. Farghal, A.E.A.: On the Performance of OCDMA/SDM PON Based on FSO under Atmospheric Turbulence and Pointing Errors. Optics and Laser Technology 114 (February): 196-203 (2019). https://doi.org/10.1016/j.optlastec.2019.01.048.

11. Fortier, T., Baumann, E.: 20 Years of Developments in Optical Frequency Comb Technology and Applications. Communications Physics, 1-16 (2019). https://doi.org/10.1038/s42005-019-0249-y.

12. Freude, W., René S., Bernd N., Marcus W., Arne J., David H., Swen K., et al.: Quality Metrics for Optical Signals:Eye Diagram, Q-Factor, OSNR, EVM and BER. 14th International Conference on Transparent Optical Networks ICTON 2012 (2012). https://doi.org/10.1109/ICTON.2012.6254380.

13. Kakaee, M.H., Saleh S., Fadhil, H.A., Siti B.A.A., Makhfudzah, M.: Development of Multi-Service (MS) for SAC-OCDMA Systems. Optics and Laser Technology 60: 49-55 (2014). https://doi.org/10.1016/j.optlastec.2014.01.002.

14. Kumawat, S., Maddila, R.K.: Optical Fiber Technology Development of ZCCC for Multimedia Service Using SAC-OCDMA Systems. Optical Fiber Technology 39 (May): 12-20 (2017). https://doi.org/10.1016/j.yofte.2017.09.015.

15. Li, R., Dang, A.: A Novel Coherent OCDMA Scheme over Atmospheric Turbulence Channels. IEEE Photonics Technology Letters 1135 (c): 1-4 (2017). https://doi.org/10.1109/LPT.2017.2652727.

16. Liu, J., Lu, Y., Changjian G.: Demonstration of Low-Cost Uplink Transmission in a Coherent OCDMA PON Using Gain-Switched Fabry - Pérot Lasers With External Injection. IEEE Photonics Technology Letters 22 (8): 583-85 (2010). https://doi.org/10.1109/LPT.2010.2042948.

17. Abdullah, M.K., Hasoon, M., Feras, N., Aljunid, S.A., Shaari, S.: Performance of OCDMA Systems with New Spectral Direct Detection ( SDD ) Technique Using Enhanced Double Weight (EDW) Code. Optics Communications 281: 4658-62 (2008). https://doi.org/10.1016/j.optcom.2008.06.029.

18. Matem, R., Aljunid, S.A., Junita, M.N., Rashidi C.B.M., Shihab Ahmed, I.: Photodetector Effects on the Performance of 2D Spectral/Spatial Code in OCDMA System. Optik - International Journal for Light and Electron Optics 178 (February): 1051-61(2019). https://doi.org/10.1016/j.ijleo.2018.10.068.

19. Menon, P.S., Ali, Z.G., Mandeep, J.S., Shaari, S.: Realization of 2-D OCDMA Network Using EDW Code. Optik - International Journal for Light and Electron Optics 123 (15): 1385-89 (2012). https://doi.org/10.1016/j.ijleo.2011.07.073.

20. Mostafa, S., Mohamed, A.A.: Performance Evaluation of SAC-OCDMA System in Free Space Optics and Optical Fiber System Based on Different Types of Codes. Wireless Personal Communications 96: 2843-61 (2017). https://doi.org/10.1007/s11277-017-4327-8.

21. Mrabet, H., Cherifi, A., Raddo, T., Dayoub, I.: A Comparative Study of Asynchronous and Synchronous OCDMA Systems. IEEE Systems Journal, 1-12 (2020). https://doi.org/10.1109/JSYST.2020.2991678.

22. Mrabet, H., Mhatli, S., Dayoub, I., Giacoumidis, E.: Performance Analysis of AO-OFDM-CDMA with Advanced 2D-Hybrid Coding for Amplifier- Free LR-PONs. IET Optoelectronics 12 (6): 293-98 (2018). https://doi.org/10.1049/iet-opt.2018.5042.

23. Nawawi, N. M., Anuar, M.S., Junita, M. N.: Cardinality Improvement of Zero Cross Correlation (ZCC) Code for OCDMA Visible Light Communication System Utilizing Catenated-OFDM Modulation Scheme. Optik - International Journal for Light and Electron Optics 170 (May): $220-25$ (2018). https://doi.org/10.1016/j.jleo.2018.05.125.

24. Painchaud, Y., Poulin, M., Morin, M., Têtu, M.: Performance of Balanced Detection in a Coherent Receiver. Optics Express 17 (5): $3659-72$ (2009). https://doi.org/10.1364/OE.17.003659.

25. Sarangal, H., Singh, A., Malhotra, J., Chaudhary, S.: A Cost Effective 100 Gbps Hybrid MDM-OCDMA-FSO Transmission System under Atmospheric Turbulences. Optical and Quantum Electronics 49 (5): 1-10 (2017). https://doi.org/10.1007/s11082-017-1019-2.

26. Schmogrow, R., Nebendahl, B., Winter, M., Josten, A., Hillerkuss, D., Koenig, S., Meyer, J., et al.: Error Vector Magnitude as a Performance Measure for Advanced Modulation Formats. IEEE Photonics Technology Letters 24 (1): 2011-13 (2012). https://doi.org/10.1109/LPT.2011.2172405.

27. Seyedzadeh, S., Pour, F., Glesk, Ivan., Kakaee, M.H. Optical Fiber Technology Variable Weight Spectral Amplitude Coding for Multiservice OCDMA Networks. Optical Fiber Technology 37: 53-60 (2017). https://doi.org/10.1016/j.yofte.2017.07.002.

28. Shang, L., Li, Y., Ma, L, Chen, J.: A Flexible and Ultra- Flat Optical Frequency Comb Generator Using a Parallel Mach - Zehnder Modulator with a Single DC Bias. Optics Communications 356: 70-73 (2015). https://doi.org/10.1016/j.optcom.2015.07.065.

Page $8 / 16$ 
29. Sheetal, A., Singh, H.: $5 \times 10$ Gbps WDM-CAP-PON Based on Frequency Comb Using OFDM with Blue LD. Optical and Quantum Electronics 50: 446 (2018). https://doi.org/10.1007/s11082-018-1703-x.

30. Shieh, W., Bao, H., Tang, Y.: Coherent Optical OFDM: Theory and Design. Optics Express 16 (2): 841-59 (2008). https://doi.org/10.1364/OE.16.000841.

31. Singh, S., Singh, S.: Performance Analysis of Spectrally Encoded Hybrid WDM-OCDMA. AEUE - International Journal of Electronics and Communications (2017). https://doi.org/10.1016/j.aeue.2017.10.003.

32. Tharek, A.O.A., Rahman, A., Aljunid, S.A.: A New Model to Enhance the QoS of Spectral Amplitude Coding-Optical Code Division Multiple Access System with OFDM Technique. Optical and Quantum Electronics (2016). https://doi.org/10.1007/s11082-016-0750-4.

33. Tithi, F.H., Majumder, S.P.: Analytical Evaluation of Combined Influence of XPM, ASE and SRS in a Raman Amplifier Based WDM System. Optik International Journal for Light and Electron Optics 208: 164076 (2020). https://doi.org/10.1016/j.ijleo.2019.164076.

34. Tran, T.T., Song, M., Song, M., Seo, D.: Highly Flat Optical Comb Generation Based on DP-MZM and Phase Modulators. Electronics Letters 55 (1): $43-45$ (2019). https://doi.org/10.1049/el.2018.6454.

35. Upadhyay, K.K., Shukla, N.K., Chaudhary, S.: A High Speed 100 Gbps MDM-SAC-OCDMA Multimode Transmission System for Short Haul Communication. Optik - International Journal for Light and Electron Optics 202: 163665 (2019). https://doi.org/10.1016/j.ijleo.2019.163665.

36. Yousif, B., Ibrahim, E.M.A., and Samra, S.: A Modified Topology Achieved in OFDM / SAC-OCDMA-Based Multi-Diagonal Code for Enhancing Spectral Efficiency. Photonic Network Communications (2018). https://doi.org/10.1007/s11107-018-0796-2.

\section{Figures}

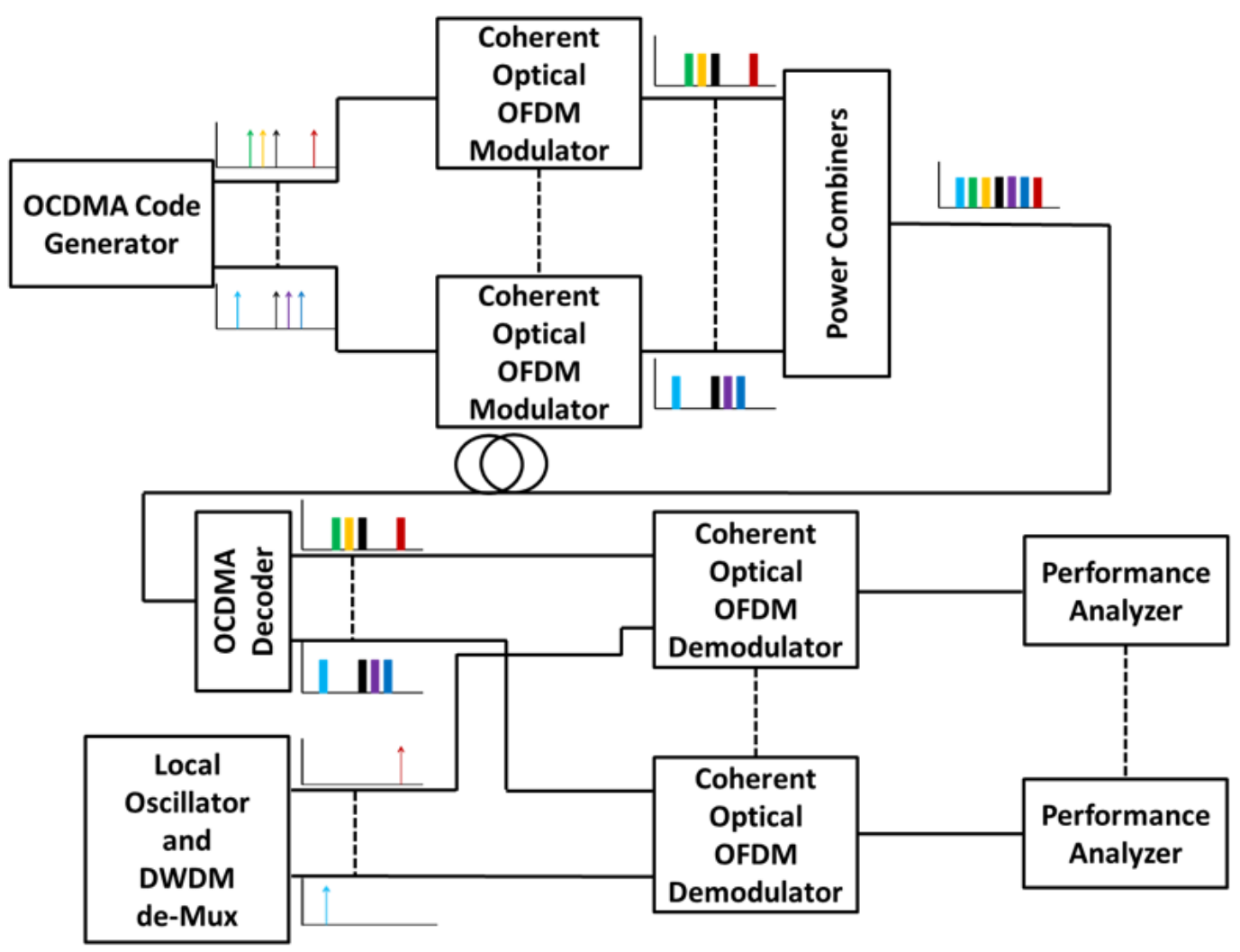

Figure 1

Block diagram of the proposed system 


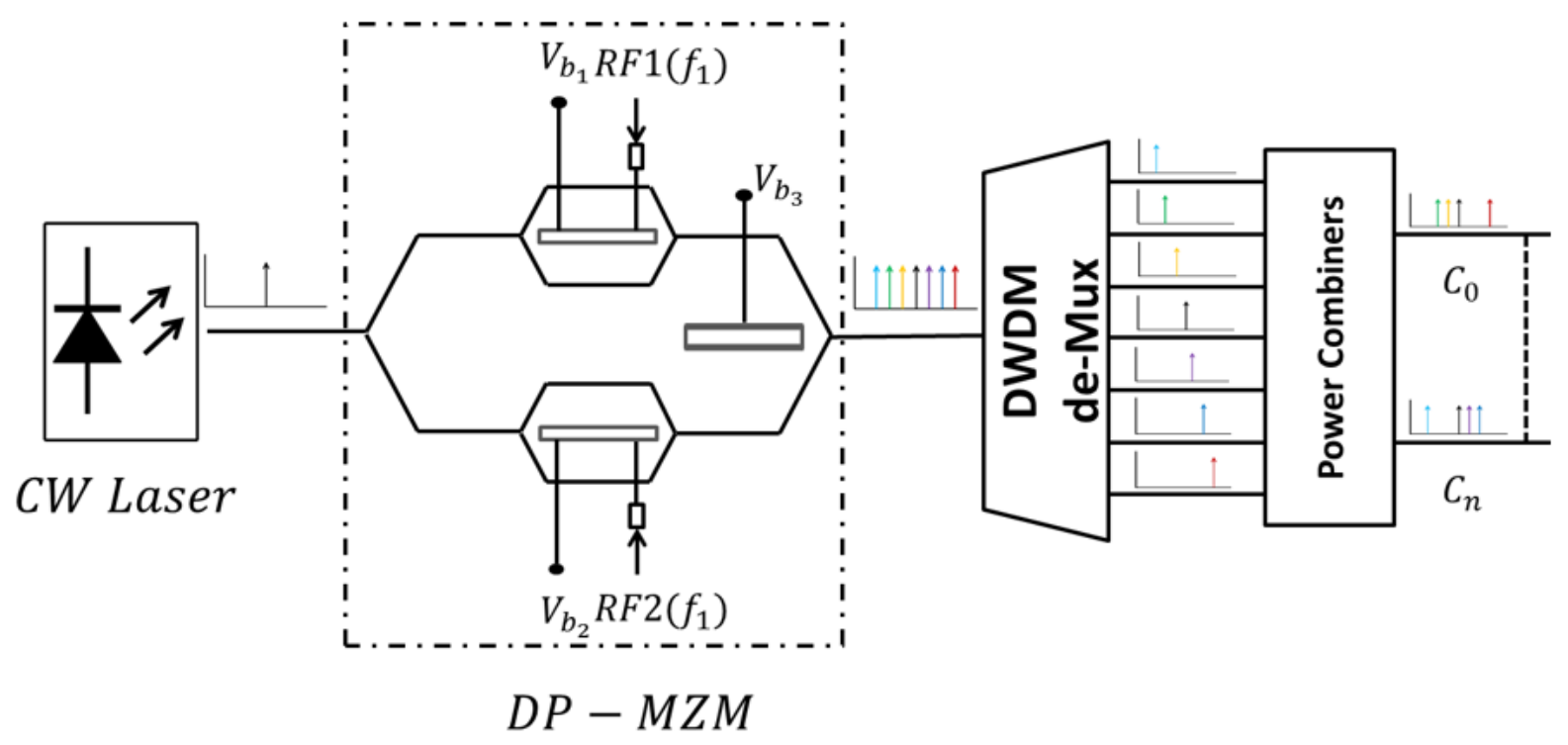

Figure 2

The OCDMA encoder uses DP-MZM, DWDM de-Mux and power combiners.

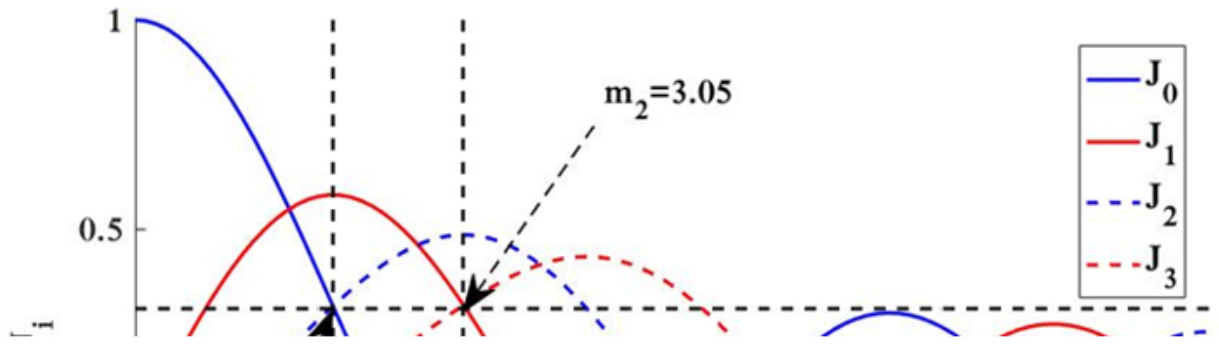

Figure 3

Bessel function of ith order of first kind. 


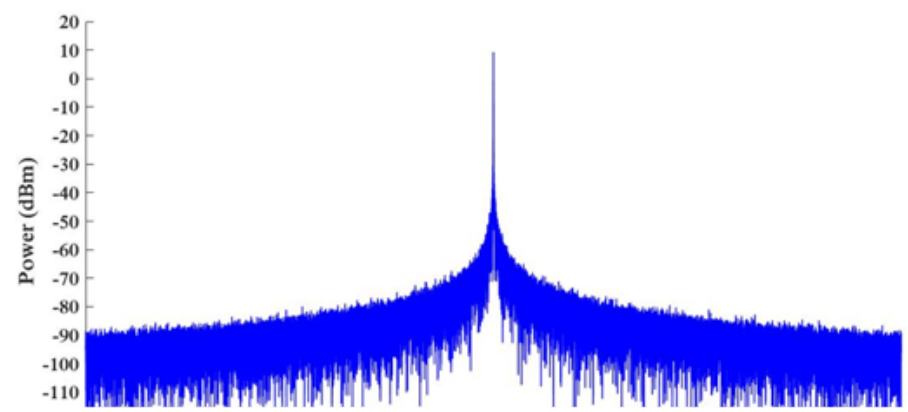

Figure 4

(a) Spectrum of the CW laser and (b) the flat OFC generator

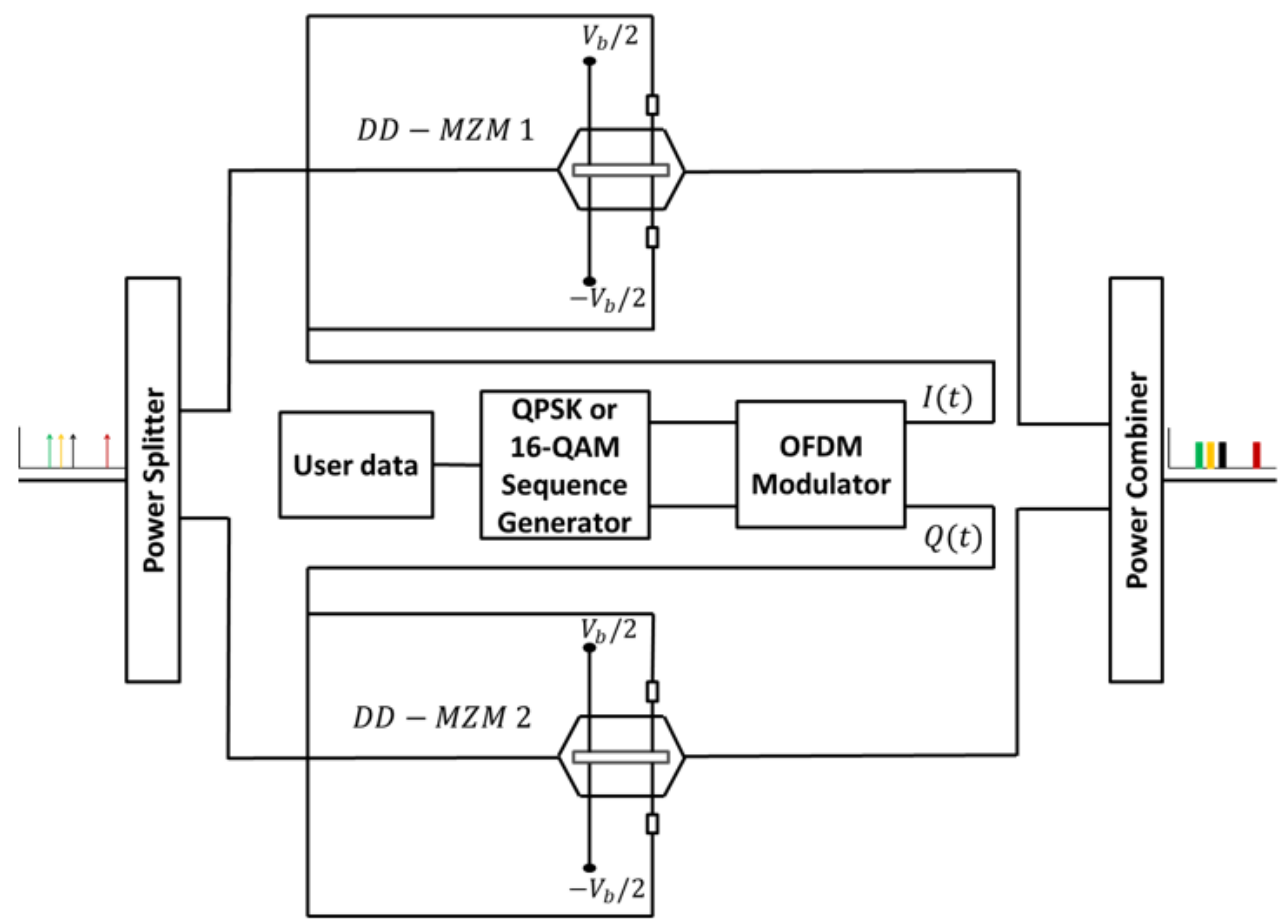

Figure 5

Coherent Optical OFDM Modulator. 


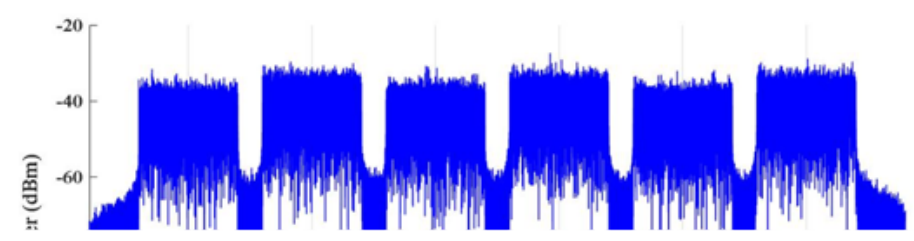

Figure 6

(a) Output spectrum of EDW and (b) RD code

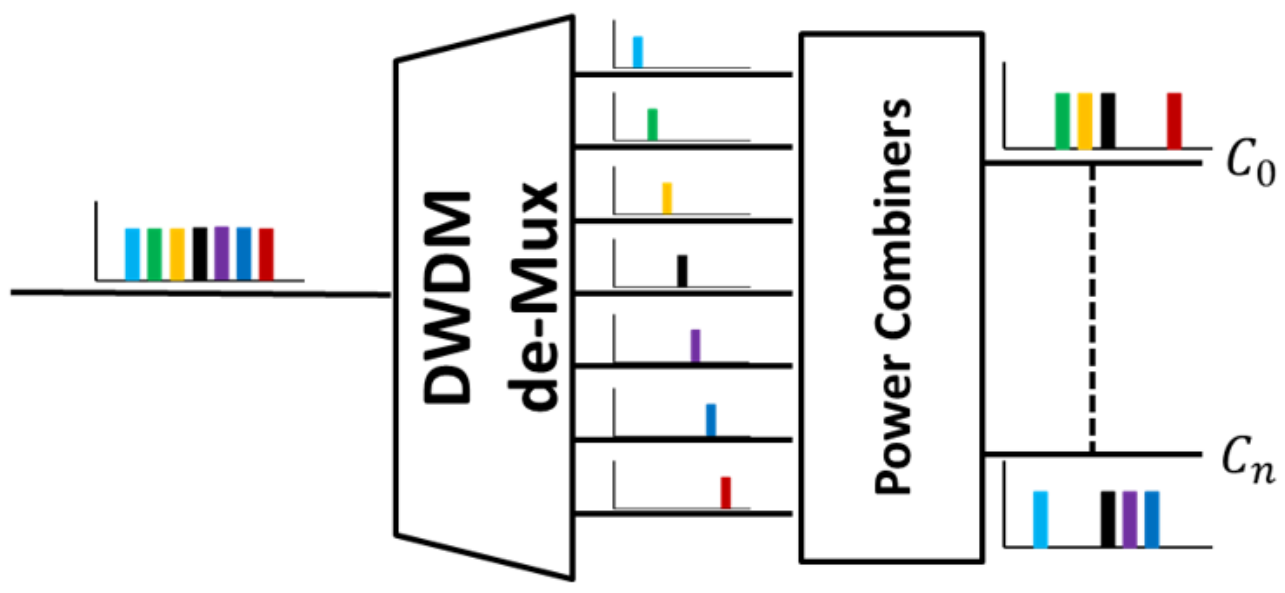

Figure 7

SAC-OCDMA decoder. 


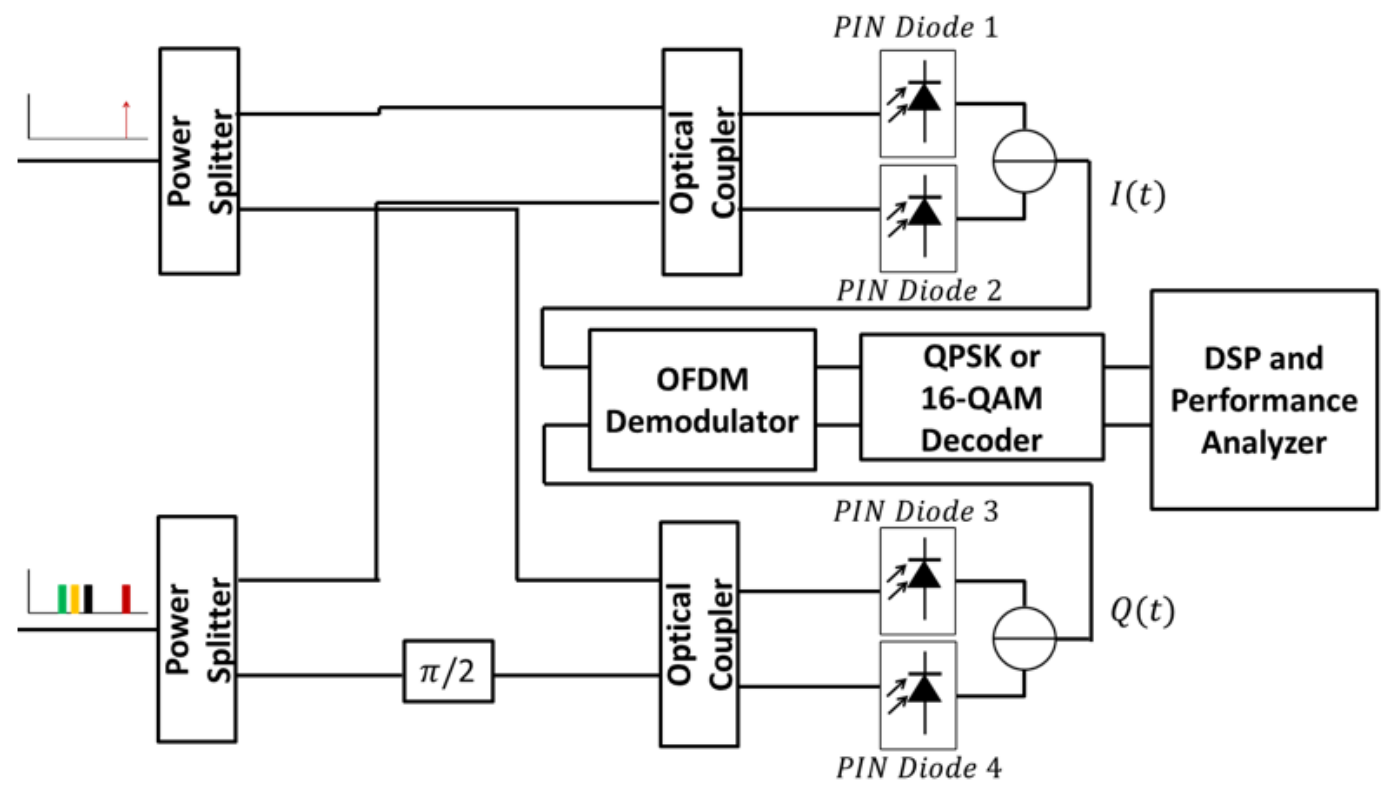

Figure 8

the receiver part for each user.

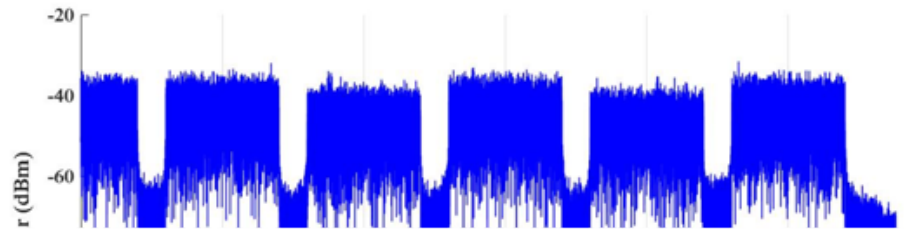

Figure 9

Received spectrums of the third user of (a) EDW code (b) and RD code. 


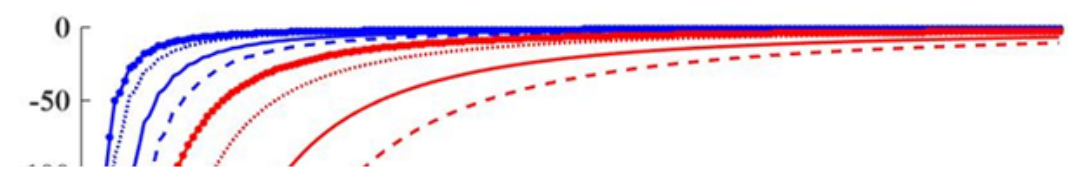

Figure 10

$\log (B E R)$ versus number of users of optical coherent detection for different code weights and different bit rates in the presence of ASE noise.

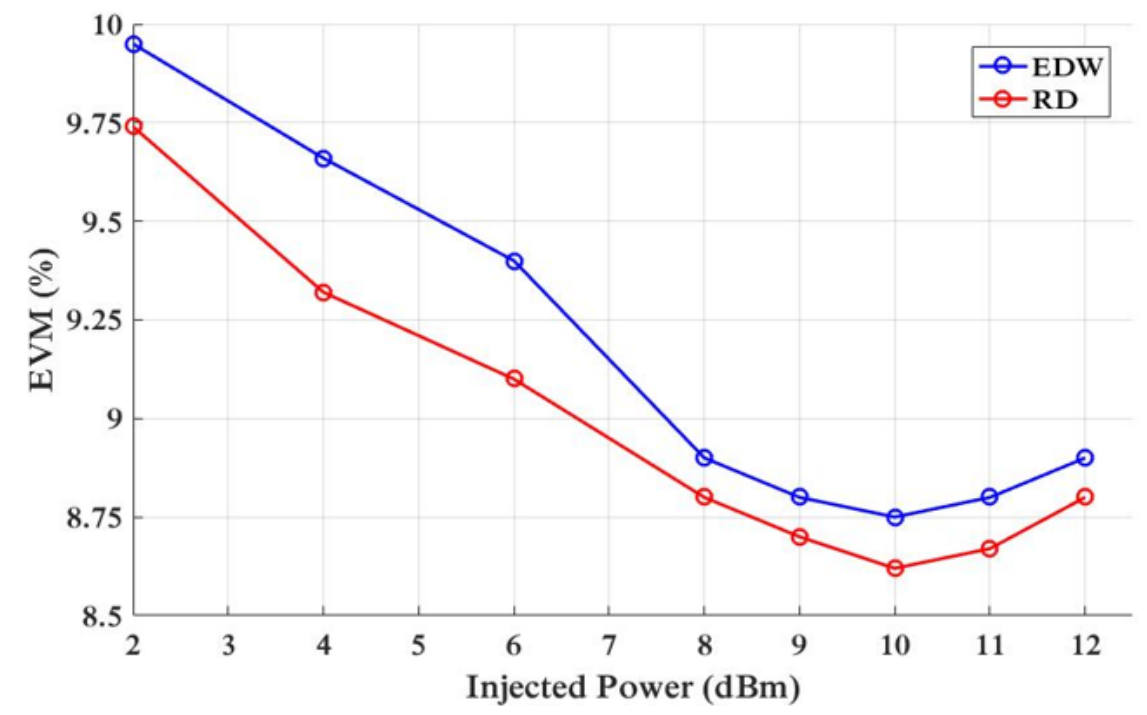

Figure 11

the EVM versus the CW laser injected power. 


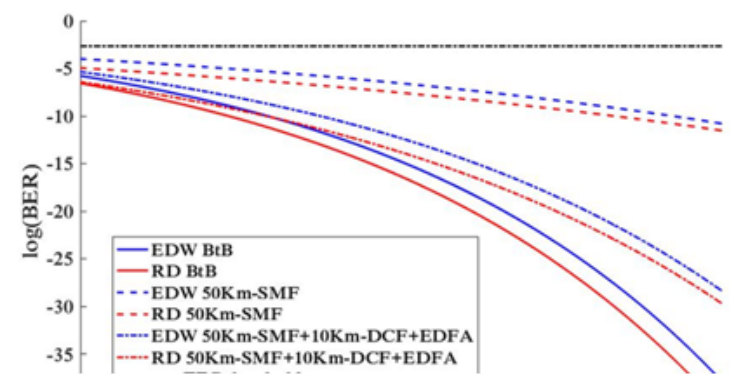

\section{Figure 12}

$\log (B E R)$ in function of OSNR for symbol rate of 10 GBd (a) using QPSK modulation, (b) using 16-QAM modulation.

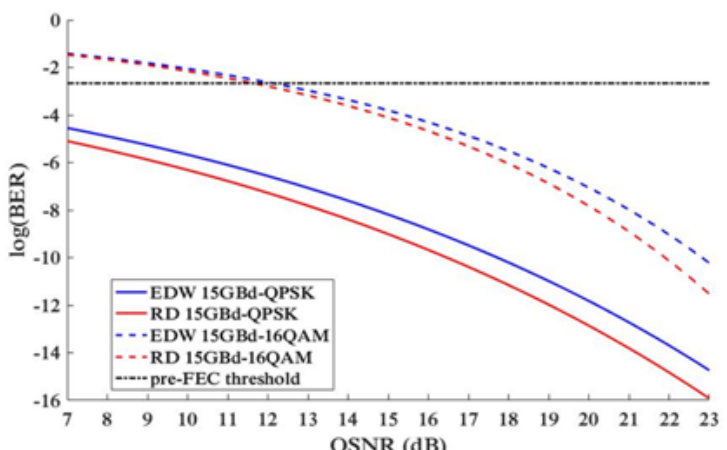

(a)

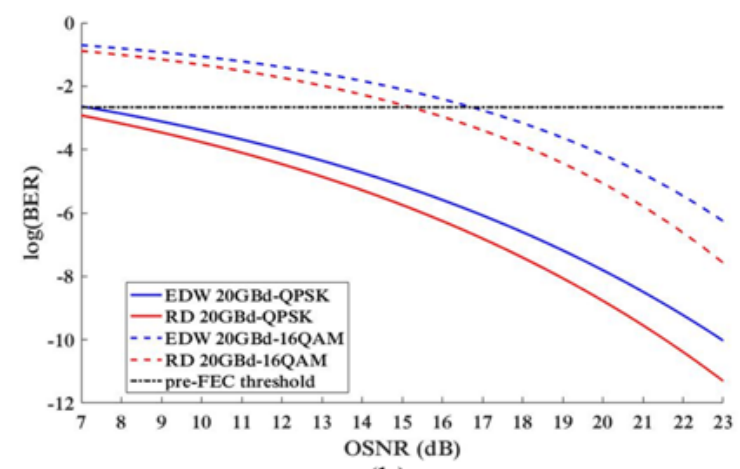

(b)

Figure 13 
$\log (\mathrm{BER})$ versus OSNR for a distance of $50 \mathrm{Km}-\mathrm{SMF}+10 \mathrm{Km}$-DCF+EDFA for different symbol rates, (a) $15 \mathrm{GBd}$, (b) 20 GBd.

\section{Figure 14}

Constellation diagrams for different distances with symbol rate of $20 \mathrm{GBd}$ 